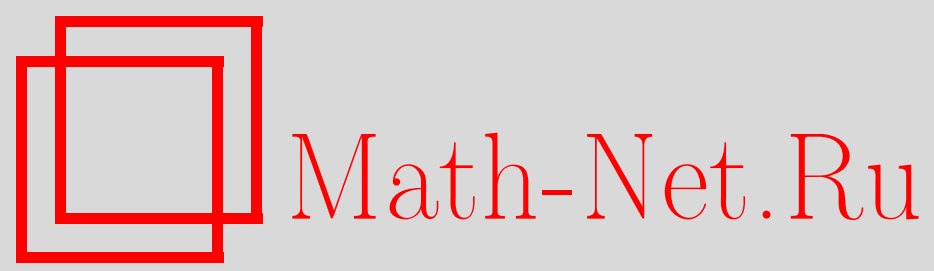

С. А. Рогонов, И. С. Солдатенко, О распределении максимума случайных величин, Нечеткие системы и мягкие вычисления, 2020, том 15, выпуск 2, 124-136

DOI: https://doi.org/10.26456/fssc74

Использование Общероссийского математического портала Math-Net.Ru подразумевает, что вы прочитали и согласны с пользовательским соглашением

http://www.mathnet.ru/rus/agreement

Параметры загрузки:

IP : 44.207 .124 .84

26 апреля 2023 г., 15:54:40 
УДК 519.8

\section{О РАСПРЕДЕЛЕНИИ МАКСИМУМА СЛУЧАЙНЫХ ВЕЛИЧИН ${ }^{1}$}

Рогонов С.А., Солдатенко И.С.

Тверской государственный университет, г. Тверь

Поступила в редакцию 01.12.2020, после переработки 16.12.2020.

В работе исследуется способ идентификации методом Монте-Карло распределений нечетких случайных величин, в параметрическом задании которых присутствует функция максимума от взвешенных случайных величин.

Ключевые слова: нечеткая случайная величина, возможность, максимум, метод Монте-Карло.

Нечеткие системы и мягкие вычисления. 2020. T. 15, № 2. С. 124-136. https://doi.org/10.26456/fssc74

\section{Введение}

Возможностная оптимизация - научное направление, лежащее на стыке двух дисциплин: молодой, активно развивающейся теории возможностей и фундаментального аппарата теории оптимизации. Теория возможностей получила в настоящее время широкую популярность благодаря востребованности инструментария, подходящего для более адекватного моделирования неопределенности, которая не всегда может быть описана формализмами теории вероятностей. Неопределенность, возникающую при попытке построения моделей, в которых задействованы человеческие суждения, процесс принятия решений, экспертные оценки и все другие области, в которых так или иначе используется человек в качестве одного из источников информации, удобней всего моделировать при помощи аппарата нечетких (возможностных) величин. Иными словами, теория возможностей более адекватно моделирует субъективную неопределенность. В свою очередь теория вероятностей как нельзя лучше подходит для моделирования неопределенности объективного типа.

Иногда возникает необходимость ввести в рассматриваемую модель неопределенность того и другого типа - в этом случае получаются задачи возможностно-вероятностной оптимизации или, как их еще называют, задачи оптимизации в условиях гибридной неопределенности. Одним из примеров подобных задач являются задачи портфельного анализа, в которых с помощью вероятностной компоненты моделируется объективная неопределенность, существующая, например, на рынке ценных бумаг, в то время как возможность необходима для задания экспертных оценок. Рассмотрим в качестве примера стоимость акций некоторой компании. Удобней задавать этот актив не просто случайной величиной, а некоторым случайным диапазоном с нечеткими границами.

${ }^{1}$ Работа выполнена при финансовой поддержке РФФИ (проект №20-01-00669). 
При решении задач портфельного анализа в условиях гибридной неопределенности прежде всего необходимо научиться снимать эти неопределенности и приводить модели к детерминированному эквивалентному виду. Для этого необходимо сначала идентифицировать возможностно-вероятностное распределение параметров модели. В настоящее время актуальной является задача исследования моделей в том случае, когда возможностные параметры этих моделей являются взаимодействующими. Взаимодействие (взаимная связанность) возможностных величин моделируется при помощи аппарата треугольных норм, используемого в определении их совместной функции распределения возможности.

Одним из способов интерпретации взаимодействия, описываемого с помощью t-норм, является агрегирование нечеткой информации. Предположим, мы имеем $n$ нечетких величин, которые необходимы для решения некоторой задачи возможностной оптимизации и заданы своими функциями распределения. Непосредственно перед тем, как приступить к решению задачи, мы должны ввести дополнительное условие, описывающее их взаимодействие (Т-связанность, где $\mathrm{T}$ - треугольная норма). Например, при $T=\min$ мы получаем минисвязанные нечеткие величины, а при слабейшей t-норме $-T_{W}$-связанные. Введение этого условия осуществляется путем (неявного) конструирования модельного множества, в рамках которого в дальнейшем решается оптимизационная задача. В работе [1], в частности, приведен пример, как можно явно сконструировать модельное множество по совокупности зараннее заданных нечетких величин, в котором все эти величины будут удовлетворять нужному свойству взаимной связанности. В данном случае треугольная норма используется в качестве механизма агрегирования нечеткой информации, осуществляемого совместной функцией распределения возможности $n$ нечетких величин, используемой при построении модельного множества с заданными характеристиками.

В дальнейшем заложенное в условии задачи взаимодействие ее параметров играет существенную роль при ее решении. Уже при построении исчисления возможностей, используемого, в частности, для нахождения функции распределения взвешенной суммы нечетких величин, мы получаем принципиально разные результаты (см., например, [2] - для минисвязанности и [3] - для взаимной $T_{W}$-связанности параметров), которые приводят к различным методам решения поставленных задач.

Применительно к задачам портфельной оптимизации в условиях гибридной неопределенности, слабейшая t-норма приводит к появлению в распределениях максимума от взвешенных случайных величин. Для дальнейшей работы с ними необходимо уметь идентифицировать подобные распределения. В настоящей статье исследуется способ идентификации подобного распределение методом Монте-Карло.

\section{1. Основные элементы теории возможностей}

Введем необходимые в дальнейшем определения и понятия из теории возможностей $[2,4-9]$.

Пусть $\Gamma$ - модельное пространство, $\gamma \in \Gamma$ - его элементы, а $\mathbb{P}(\Gamma)$ - множество всех подмножеств множества $\Gamma, \mathbb{E}^{1}-$ числовая прямая.

Определение 1. Возможностная мера $\pi: \mathbb{P}(\Gamma) \rightarrow E^{1}-$ есть функция множества, обладающая следующими свойствами: 
1. $\pi(\oslash)=0, \pi(\Gamma)=1$,

2. $\pi\left(\bigcup_{i \in I} A_{i}\right)=\sup _{i \in I} \pi\left(A_{i}\right), \forall A_{i} \in \mathbb{P}(\Gamma), \forall I$.

Определение 2. Тройка $(\Gamma, \mathbb{P}(\Gamma), \pi)$ называется возможностным пространством.

Определение 3. Возможностной (нечеткой) величиной называется вещественная функиия

$$
A(\cdot): \Gamma \rightarrow \mathbb{E}^{1},
$$

характеризующаяся распределением возможностей $\mu_{A}(x)$ :

$$
\mu_{A}(x)=\pi\{\gamma \in \Gamma: A(\gamma)=x\}, \forall x \in \mathbb{E}^{1},
$$

где $\mu_{A}(x)$ - возможность того, что $A$ может принять значение $x$.

Определение 4. Носителем возможностной величины $A$ называется четкое подмножество:

$$
\operatorname{supp}(A)=\left\{x \mid \mu_{A}(x)>0\right\}, x \in \mathbb{E}^{1} .
$$

Определение 5. Модальным значением возможностной величины $А$ называется следующее четкое подмножество:

$$
\left\{x \mid \mu_{A}(x)=1\right\}, x \in \mathbb{E}^{1} .
$$

Определение 6. Для любой возможностной переменной $A$ и любого $\alpha \in[0,1]$, $\alpha$-уровневым множеством $A(\alpha)$ называется:

- $A(\alpha)=\left\{x \in \mathbb{E}^{1} \mid \mu_{A}(x) \geq \alpha\right\}, \partial л я \alpha \in(0,1]$,

- $A(\alpha)=\operatorname{cl}(\operatorname{supp}(A)), \partial л я \alpha=0$,

где $\operatorname{cl}(\operatorname{supp}(A))$ - замыкание носителя возможностной переменной $A$.

Определение 7. Возможностная переменная А называется выпуклой, если ее функиия распределения является квазивогнутой:

$$
\mu_{A}\left(\lambda x_{1}+(1-\lambda) x_{2}\right) \geq \min \left\{\mu_{A}\left(x_{1}\right), \mu_{A}\left(x_{2}\right)\right\}, \lambda \in[0,1], x_{1}, x_{2} \in \mathbb{E}^{1} .
$$

Возможностные переменные на числовой прямой, характеризующиеся строго унимодальными, квазивогнутыми, полунепрерывными сверху функциями распределения и ограниченными носителями, называются нечеткими числами.

Для моделирования нечетких чисел часто используются распределения $(L, R)$-типа $[10,11]$.

Определение 8. $(L, R)$ - бункииями (функциями представления формы или просто формами) будем называть невозрастающие, полунепрерывные сверху, определенные на неотрицательной части числовой прлмой функции, обладающие следующими свойствами:

1. $L(0)=R(0)=1$,

2. $L(t), R(t)<1, \quad \forall t>0$, 
3. $\lim _{t \rightarrow \infty} L(t)=\lim _{t \rightarrow \infty} R(t)=0$.

Определение 9. Возможностная величина А называется возможностной величиной $(L, R)$-типа, если ее распределение имеет вид:

$$
\mu_{A}(x)= \begin{cases}L\left(\frac{a-x}{\alpha}\right), & x<a, \\ 1, & a \leq x \leq b, \\ R\left(\frac{x-b}{\beta}\right), & x>b .\end{cases}
$$

Здесь [a, b] - есть интервал толерантности $\mathrm{A}, \mathrm{a}$ и $\mathrm{b}$ - соответственно, нижнее и верхнее модальные значения, $\alpha$ и $\beta$ - коэффициенты нечеткости возможностной величины. Если $a=b$, то получаем нечеткое число, которое будем обозначать как $A=(a, \alpha, \beta)_{L R}$.

Пример 1. Если в качестве левой и правой форм взять кусочно-линейную функцию $L(x)=R(x)=\max \{0,1-x\}, x \geq 0$, то для случая нечетких чисел мы получаем так называемые триангулярные нечеткие числа.

Распределения возможностных величин $(L, R)$-типа удобны тем, что они являются параметризованными, что позволяет в ряде случаев сводить выполнение основных арифметических операций над возможностными величинами к выполнению этих операций над параметрами.

Пусть также $(\Omega, B, P)$ есть вероятностное пространство. Дадим понятие нечеткой случайной величины $[2,4]$.

Определение 10. Нечеткал случайная величина $Y(\omega, \gamma)$ есть вещественнал функиия $Y: \Omega \times \Gamma \rightarrow \mathbb{E}^{1} \sigma$-измеримая для каждого фиксированного $\gamma$, а функиия

$$
\mu_{Y}(\omega, t)=\pi\{\gamma \in \Gamma: Y(\omega, \gamma)=t\}, \forall t \in \mathbb{E}^{1}
$$

называется ее функиией распределения.

Из определения 10 следует, что функция распределения нечеткой случайной величины зависит от случайного параметра, т. е. является случайной функцией.

\section{2. Задача портфельной оптимизации в условиях гибридной неопреде- ленности}

В задаче портфельного анализа, построенной в соответствии с классическим подходом Марковица [12], одной из главных составляющих является функция доходности инвестиционного портфеля. Будем представлять ее нечеткой случайной функцией:

$$
R_{p}(w, \omega, \gamma)=\sum_{i=1}^{n} R_{i}(\omega, \gamma) w_{i}
$$

которая является линейной функцией долей капитала $w=\left(w_{1}, w_{2}, \ldots, w_{n}\right)$ в инвестиционном портфеле. Доходности отдельных финансовых активов $R_{i}(\omega, \gamma)$ будем моделировать с помощью сдвиг-масштабного представления [4]:

$$
R_{i}(\omega, \gamma)=a_{i}(\omega)+\sigma_{i}(\omega) Z_{i}(\gamma)
$$


Далее будем предполагать, что нечеткие величины $Z_{i}(\gamma)=\left[\underline{m}_{i}, \bar{m}_{i}, \underline{d}_{i}, \bar{d}_{i}\right]_{L R}$ взаимно $T_{W}$-связаны, то есть их взаимодействие описывается слабейшей $t$-нормой $T_{W}$, a $a_{i}(\omega)$ и $\sigma_{i}(\omega)$ - коэффициенты сдвига и масштаба - независимые одинаково распределенные случайные величины, имеющие нормальное распределение.

В этом случае возможностное распределение доходности портфеля принимает вид:

$$
R_{p}(w, \omega, \gamma)=\left[\underline{m}_{R_{p}}(w, \omega), \bar{m}_{R_{p}}(w, \omega), \underline{d}_{R_{p}}(w, \omega), \bar{d}_{R_{p}}(w, \omega)\right]_{L R}
$$

где

$$
\begin{array}{cc}
\underline{m}_{R_{p}}(w, \omega)=\sum_{i=1}^{n}\left(a_{i}(\omega)+\sigma_{i}(\omega) \underline{m}_{i}\right) w_{i}, & \bar{m}_{R_{p}}(w, \omega)=\sum_{i=1}^{n}\left(a_{i}(\omega)+\sigma_{i}(\omega) \bar{m}_{i}\right) w_{i}, \\
\underline{d}_{R_{p}}(w, \omega)=\max _{i=1 \ldots n}\left\{\sigma_{i}(\omega) \underline{d}_{i} w_{i}\right\}, & \bar{d}_{R_{p}}(w, \omega)=\max _{i=1 \ldots n}\left\{\sigma_{i}(\omega) \bar{d}_{i} w_{i}\right\} .
\end{array}
$$

Исследуем распределение случайных величин $\max _{i=1 \ldots n}\left\{\sigma_{i}(\omega) \underline{d}_{i} w_{i}\right\}$ и $\max _{i=1 \ldots n}\left\{\sigma_{i}(\omega) \bar{d}_{i} w_{i}\right\}$.

\section{3. Идентификация распределения максимума случайных величин}

Переопределим, без потери общности, случайные величины $\max _{i=1 \ldots n}\left\{\sigma_{i}(\omega) \underline{d}_{i} w_{i}\right\}$ и $\max _{i=1 \ldots n}\left\{\sigma_{i}(\omega) \bar{d}_{i} w_{i}\right\}$ следующим образом:

$$
Q=\max _{i=1 \ldots n}\left\{c_{i} X_{i}\right\}
$$

где

$$
X_{i} \sim N\left(\mu_{i}, \sigma_{i}^{2}\right)
$$

Покажем, что умножение нормально распределённой случайной величины на постоянный коэффициент эквивалентно нормально распределённой случайной величине с точностью до параметров:

$$
c_{i} X_{i}=Y_{i} \sim N\left(c_{i} \mu_{i}, c_{i}^{2} \sigma_{i}^{2}\right)
$$

В соответствии с полученным результатом переопределим случаюную величину из уравнения 2 :

$$
Q=\max _{i=1 \ldots n}\left\{Y_{i}\right\}
$$

На основании полученного выражения задача о взвешенном максимуме для случая нормально распределённых случайных величин свелась к задаче обычного максимума.

\section{4. Моделирование методом Монте-Карло}

Эффективным способом получить представление о распределении случайной величины заданной нетривиальным выражением, можно с помощью численного моделирования. В данной работе применён метод Монте-Карло, реализация на языке Python. 


\section{1 Преобразование Бокса - Мюллера}

Для генерации нормально распределённой случайно величины используем метод полярных координат, предложенный Джорджем Боксом, Мервином Мюллером и Джорджем Марсальей в 1958 году. Данный метод преобразует две независимые равномерно распределённые случайные величины в две независимые нормально распределённые величины. Дадим формальное описание метода:

$$
\begin{gathered}
A \sim U(0,1) ; B \sim U(0,1) \\
Z_{0}=\sqrt{-2 \ln (A)} \cos (2 \pi B) \sim N(0,1) \\
Z_{1}=\sqrt{-2 \ln (A)} \sin (2 \pi B) \sim N(0,1)
\end{gathered}
$$

Так как в данной работе не преследуется цель генерации случайных величин максимально эффективным способом, в силу удобства дальнейшей реализации на Python остановимся на одной нормальной величине вместо двух. Ниже представлена реализация на языке Python использованная в данной работе:

\section{Листинг 1: Преобразование Бокса - Мюллера}

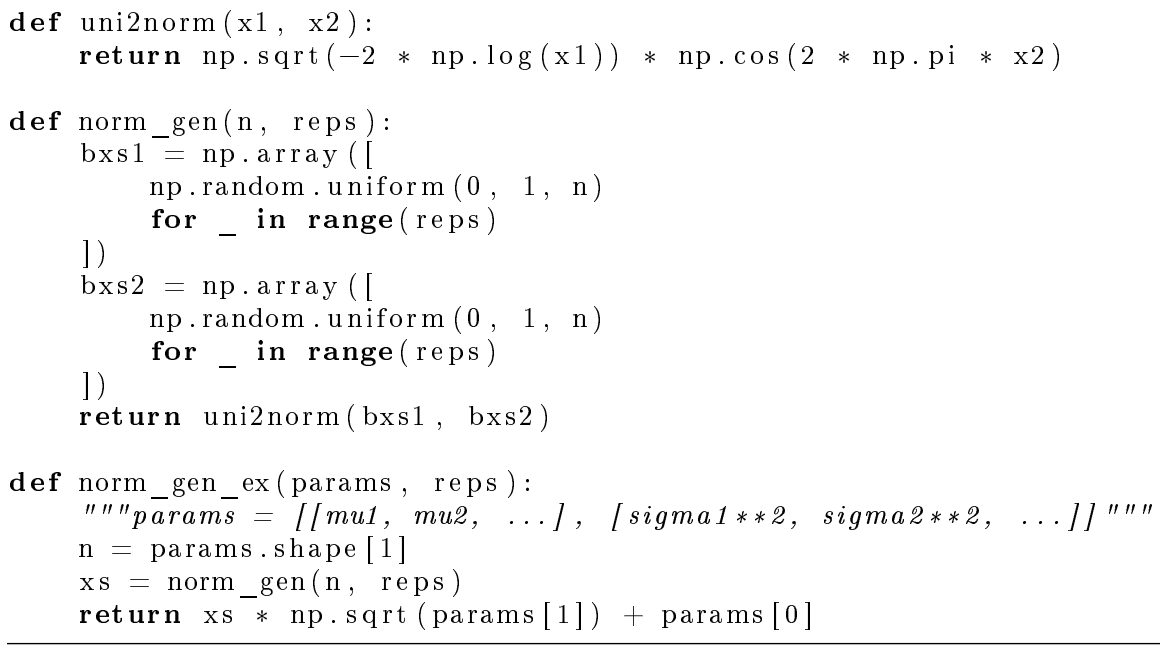

\section{2 Моделирование максимума}

Непосредственно рассмотрим распределение максимума из множества независимых нормально распределённых случайных величин. Для этого были смоделированы три ситуации:

1. Максимум из 20 нормальных величин с параметрами $\mu=0, \sigma^{2}=1$ 


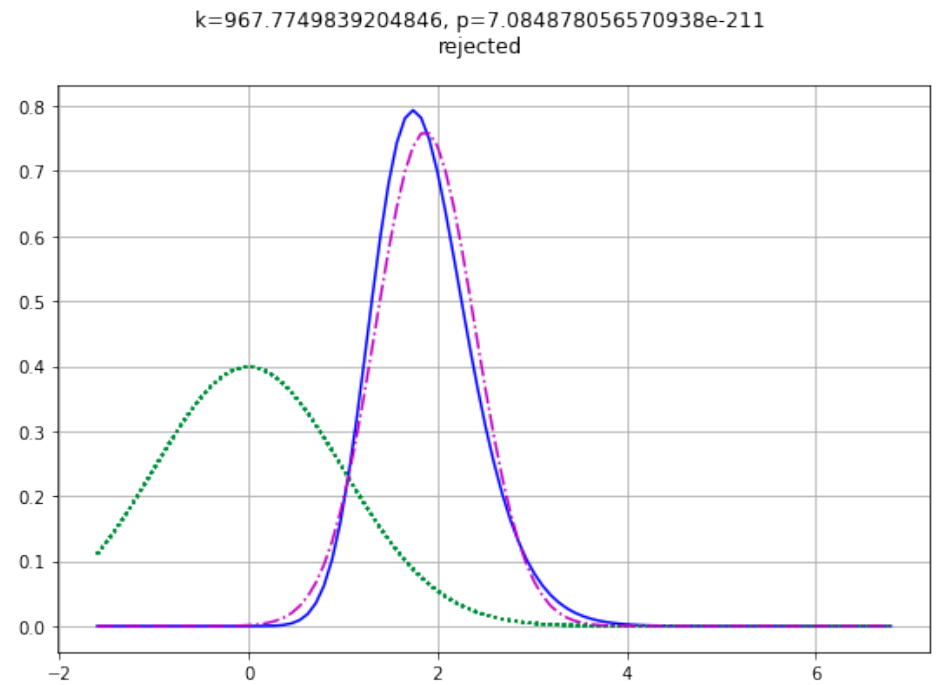

2. Максимум из 20 нормальных величин среди которых:

- 19 с параметрами $\mu=-5, \sigma^{2}=1$

- одно с параметрами $\mu=5, \sigma^{2}=1$

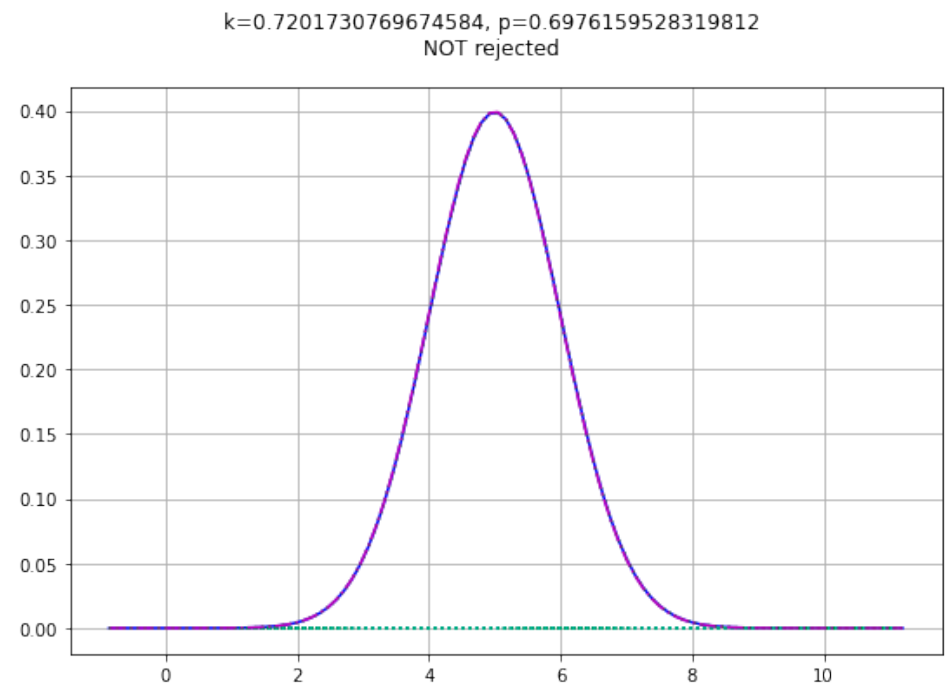

3. Две нормальные величины с параметрами

- первое $\mu=0, \sigma^{2}=1$

- второе $\mu=0.60, \sigma^{2}=10$ 


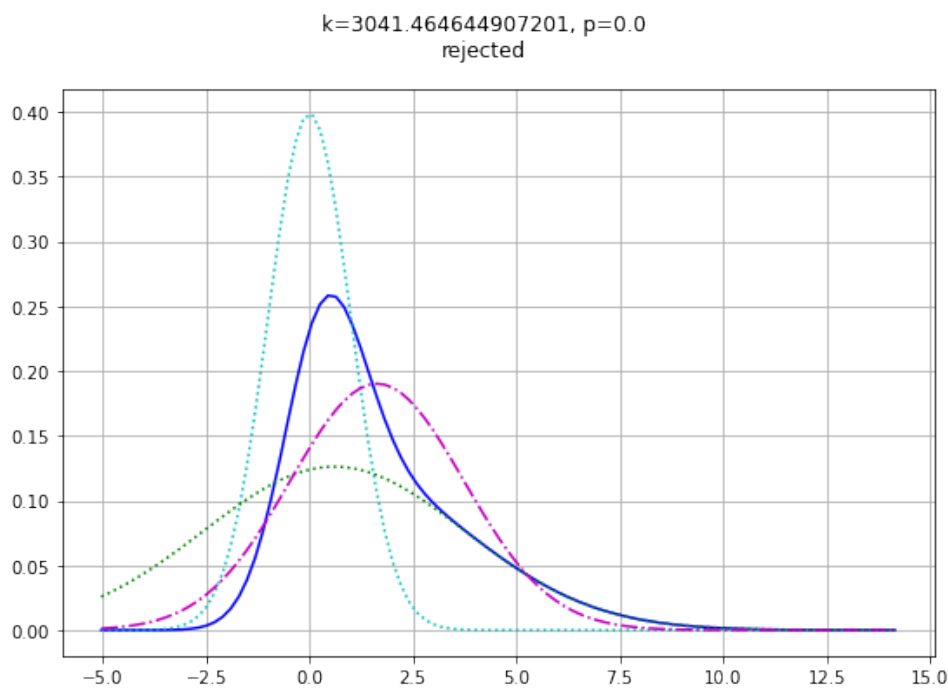

На всех изображениях использована следующая легенда:

- Точечный пунктир светло-голубого и зелёного цветов - исходные распределения нормальных величин.

- Синяя сплошная - смоделированный максимум.

- Розовый пунктир с точкой - попытка аппроксимировать полученное распределение некоторым нормальным распределением на основании среднего арифметического значения и стандартного отклонения для смоделированного распределения.

Кроме того для каждого построения рассчитан критерий Пирсона (p-value) для проверки распределения на нормальность. (D'Agostino, R. and Pearson, E. S. (1973), "Tests for departure from normality", Biometrika, 60, 613-622). Данный тест пройден только на одном моделировании из трёх, а именно втором.

\section{5. Анализ результатов моделирования}

Моделирование показало вариативное поведение максимума для разных комбинаций нормальных величин.

\section{1 Визуальный анализ}

Для множества из 20 независимых нормально распределённых величин с одинаковыми параметрами максимум ведёт себя как и ожидалось, а именно смещён относительно мат. ожидания нормальных величин в направлении положительной бесконечности. Неожиданным, но объяснимым качеством максимума является также его асимметричность - короткое левое и длинное правое плечи, так как левое плечо подпирается 20ю максимумами плотности вероятности нормального распределения, а правое плечо плавно уходит в бесконечность. Очевидно, что в силу асимметричности распределения, случайная величина определяемая 
как максимум из 20 случайных независимых нормально распределённых случайных величин не является нормально распределённой величиной, a потscipy.stats import normaltestoмy была отвергнута критерием Пирсона с надёжностью в 211 порядков величины p-value.

Для множества из 20 независимых нормально распределённых величин из которых мат. ожидание 19ти равно -5, а мат. ожидание последней равно 5, очевидно вырождение максимума в единственную случайную величину с наибольшим мат ожиданием. Разница между мат. ожиданиями столь велика, что 19 случайных величин из 20 себя никак не проявляют. Нулевая гипотеза надёжно принята критерием Пирсона, максимум достоверно описывается нормальным распределением. На графике синяя сплошная совпадает с розовой пунктир точкой и с невидимым на их фоне точечным пунктиром нормально распределённой случайной величины с мат. ожиданием равным 5 .

Для множества из двух независимых нормально распределённых величин с близким мат. ожиданием, но сильно различающейся дисперсией наиболее очевидно нетривиальное поведение максимума. Экстремально пологое правое плечо по сравнению с левым. Такое распределение ни в каком приближении нельзя считать нормально распределённым.

\section{2 Аналитический подход}

Пользуясь выражением 3 выпишем функцию распределения случайной величины $Q$ :

$$
F_{Q}(x)=P\left[\left(Y_{1} \leq x\right) \cap\left(Y_{2} \leq x\right) \cap \ldots\right]
$$

Так как все случайные нормально распределённые величины $Y_{i}$ независимы, то выражение 5 раскрывается в произведение:

$$
P\left[\left(Y_{1} \leq x\right) \cap\left(Y_{2} \leq x\right) \cap \ldots\right]=\prod_{i} P\left(Y_{i} \leq y\right)=\prod_{i} F_{Y_{i}}(x)
$$

Следовательно является возможным точное аналитическое описание функции распределения максимума из множества независимых нормальных случайных величин. На всех графиках моделирования методом Монте-Карло теоретическое предсказание совпало с синей сплошной. Ниже для сравнения даны два примера моделирования: слева построение по 20000 сгенерированных значений максимума, справа всего по 200. Красный график - построение путём моделирования, синий - путём теоретического предсказания.

Подкрепим наше утверждение о форме функции распределения случайной величины $Q$ расстоянием Кульбака - Лейблера между эмпирической и теоретической моделями. Видим, что с увеличением выборки расстояние Кульбака Лейблера падает практически до нуля. 


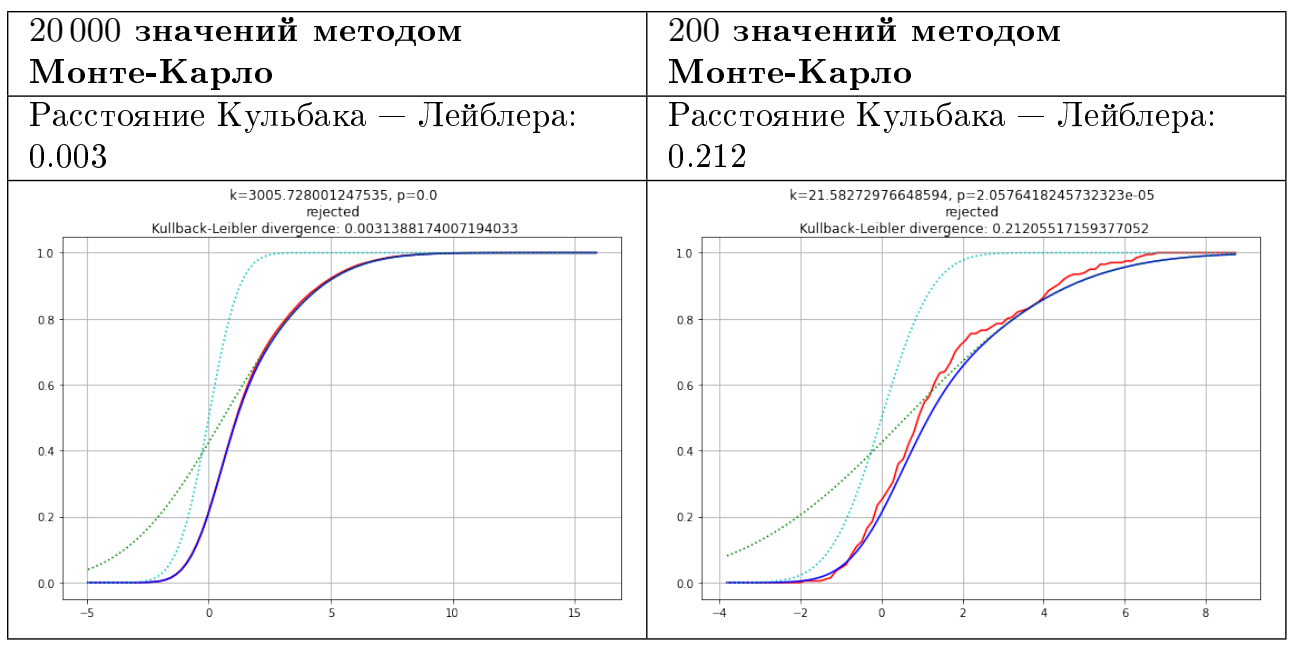

\section{Заключение}

В статье исследован способ идентификации методом Монте-Карло распределений нечетких случайных величин, в параметрическом задании которых присутствует функция максимума от взвешенных случайных величин.

\section{Список литературы}

[1] Rao M.B., Rashed A. Some comments on fuzzy variables // Fuzzy Sets and Systems. 1981. Vol. 6. Pp. 285-292.

[2] Yazenin A., Wagenknecht M. Possibilistic Optimization. A Measure-Based Approach. Cotbus, Germany: Brandenburgische Technische Universitat, 1996. $133 \mathrm{p}$.

[3] Солдатенко И.С. О взвешенной сумме взаимно Тw-связанных нечетких величин // Вестник ТвГУ. Серия: Прикладная математика. 2007. № 4. С. 63-77.

[4] Язенин А.В. Основные понятия теории возможностей. Математический аппарат для принятия решений в условиях гибридной неопределенности. М.: Физматлит, 2016. 144 с.

[5] Feng Y., Hu L., Shu H. The variance and covariance of fuzzy random variables and their applications // Fuzzy Sets and Systems. 2001. Vol. 120, № 3. Pp. 487497. https://doi.org/10.1016/S0165-0114(99)00060-3

[6] Дюбуа Д., Прад А. Теория возможностей. М.: Радио и связь, 1990.

[7] Yazenin A.V. On the problem of possibilistic optimization // Fuzzy Sets and Systems. 1996. Vol. 81. Pp. 133-140.

[8] Nahmias S. Fuzzy variables in a random environment // Advances in Fuzzy Set Theory and Applications. Eds. by M.M. Gupta, R.K. Ragade, R.R. Yager. Amsterdam: North-Holland, 1979. Pp. 165-180.

[9] Nguyen H.T., Walker E.A. A first cours in fuzzy logic. CRC Press, 1997. 
[10] Dubois D., Prade H. Fuzzy Sets and Systems: Theory and Applications. New York: Academic Press, 1980.

[11] Dubois D., Prade H. Fuzzy numbers: an overview // Analysis of Fuzzy Information. Ed. by J. Bezdek. Vol. 2. Boca Raton: CRC-Press, 1988. Pp. 339.

[12] Markowitz H. Portfolio Selection // The Journal of Finance. 1952. Vol. 7, № 1. Pp. 77-91. https://doi.org/10.2307/2975974

\section{Образец цитирования}

Рогонов С.А., Солдатенко И.С. О распределении максимума случайных величин // Нечеткие системы и мягкие вычисления. 2020. Т. 15, № 2. С. 124-136. https://doi.org/10.26456/fssc74

\section{Сведения об авторах}

\section{1. Рогонов Степан Алексеевич}

аспирант кафедры информационных технологий Тверского государственного университета.

Россия, 170100, г. Тверь, ул. Желябова, д. З3, ТвГУ.

\section{2. Солдатенко Илья Сергеевич}

доцент кафедры информационных технологий Тверского государственного университета.

Россия, 170100, г. Тверь, ул. Желябова, д. 33, ТвГУ. E-mail: soldis@tversu.ru 


\title{
ON DISTRIBUTION OF A MAXIMUM OF RANDOM VARIABLES
}

\author{
Rogonov Stepan Alekseyevich \\ PhD student at Information Technologies department, \\ Tver State University \\ Russia, 170100, Tver, 33 Zhelyabova str., TvSU.
}

Soldatenko Ilia Sergeyevich

Associate Professor in the Department of Information Technologies, Tver State University

Russia, 170100, Tver, Zhelyabova str., 33, TverSU.

E-mail:soldis@tversu.ru

Received 01.12.2020, revised 16.12.2020

In this paper, we study a method for identifying by the Monte Carlo method distributions of fuzzy random variables, in the parametric setting of which there is a maximum function of weighted random variables.

Keywords: fuzzy random variable, possibility, maximum, Monte Carlo method.

\section{Citation}

Rogonov S.A., Soldatenko I.S., "On distribution of a maximum of random variables", Nechetkie Sistemy $i$ Myagkie Vychisleniya [Fuzzy Systems and Soft Computing], 15:2 (2020), 124-136(in Russian). https://doi.org/10.26456/fssc74

\section{References}

[1] Rao M.B., Rashed A., "Some comments on fuzzy variables", Fuzzy Sets and Systems, 6 (1981), 285-292 (in Russian).

[2] Yazenin A., Wagenknecht M., Possibilistic Optimization. A Measure-Based Approach, Brandenburgische Technische Universitat, Cotbus, Germany, 1996, $133 \mathrm{pp}$.

[3] Soldatenko I.S., "On the weighted sum of mutually Tw-related fuzzy variables", Vestnik TvGU. Seriya: Prikladnaya Matematika [Herald of Tver State University. Series: Applied Mathematics], 2007, № 4, 63-77 (in Russian).

[4] Yazenin A.V., Osnovnye ponyatiya teorii vozmozhnostej. Matematicheskij apparat dlya prinyatiya reshenij $v$ usloviyakh gibridnoj neopredelennosti [Basic concepts of the theory of possibilities. Mathematical decision-making apparatus in a hybrid uncertainty], Fizmatlit Publ., Moscow, 2016 (in Russian), 144 pp.

[5] Feng Y., Hu L., Shu H., "The variance and covariance of fuzzy random variables and their applications", Fuzzy Sets and Systems, 120:3 (2001), 487-497, https://doi.org/10.1016/S0165-0114(99)00060-3.

[6] Dubois D., Prade H., Theorie des Possibilites, Application a la Representation des Connaissances en Informatique, Masson, Paris, 1988. 
[7] Yazenin A.V., "On the problem of possibilistic optimization", Fuzzy Sets and Systems, 81 (1996), 133-140.

[8] Nahmias S., "Fuzzy variables in a random environment", Advances in Fuzzy Set Theory and Applications, eds. M.M. Gupta, R.K. Ragade, R.R. Yager, NorthHolland, Amsterdam, 1979, 165-180.

[9] Nguyen H.T., Walker E.A., A first cours in fuzzy logic, CRC Press, 1997.

[10] Dubois D., Prade H., Fuzzy Sets and Systems: Theory and Applications, Academic Press, New York, 1980.

[11] Dubois D., Prade H., "Fuzzy numbers: an overview", Analysis of Fuzzy Information. V. 2, ed. J. Bezdek, CRC-Press, Boca Raton, 1988, 3-39.

[12] Markowitz H., "Portfolio Selection", The Journal of Finance, 7:1 (1952), 77-91, https://doi.org/10.2307/2975974. 